\title{
Compact Reflectometers for a Wideband Microwave Breast Cancer Detection System
}

\author{
Marek E. Bialkowski, Fellow, IEEE, Norhudah Seman, Student Member, IEEE, Amin Abbosh \\ and Wee Chang Khor, Student Member, IEEE \\ School of Information Technology and Electrical Engineering, The University of Queensland, St Lucia, \\ QLD, 4067 Australia
}

\begin{abstract}
The design of compact wideband microwave reflectometers for the purpose of inclusion in a breast cancer detection system is presented. In this system, a wideband frequency source is used to synthesize a narrow pulse via the step-frequency synthesis method. The reflectometer undertakes measurements in the frequency domain and the collected data is transformed to the time/space domain using IFFT. In order to accomplish reflection coefficient measurements over a large frequency band, compact wideband couplers and power dividers are used to form the reflectometer. Two compact six-port reflectometer configurations are investigated. One uses the Lange coupler and the Gysel power divider and the other one employs a $3 \mathrm{~dB}$ slot-coupled microstrip coupler and a 2-stage Wilkinson power divider. The reflectometer employing the slot-coupled coupler and the Wilkinson divider provides a wider operational bandwidth, as shown by simulation results performed with Agilent ADS.
\end{abstract}

Index Terms-Cancer detection, microwave imaging, experimental verification, near field.

\section{INTRODUCTION}

Breast cancer is the most common cancer diagnosed in women in various parts of the world. In Australia alone, it is estimated that one in eleven Australian women will develop breast cancer at some stage in their life [1].

Early detection and effective treatment is the only way to reduce the mortality rate due to breast cancer. Currently the primary method for breast screening is X-ray mammography [2], [3].

X-ray mammography has saved many lives, but the technology still produces a relativity high number of false negative and false positive diagnoses. There is also health concern related to exposure to ionizing radiation [3]. These factors have generated interest in alternative approaches of breast cancer detection that feature various degree of success. For example ultrasound is used clinically to discover whether a lesion detected in a mammogram is a liquid cyst or a solid tumour [2]. In addition, Magnetic Resonance Imaging has been shown to be a very useful screening tool, but is very expensive and not portable [4].

Recently, microwave imaging has been proposed as a

Manuscript received April 11, 2006. This work was supported in part by the Australian Research Council under Grants DP0449996 and DP0450118. viable alternative to X-ray mammography. Microwave imaging system is essentially "breast tissue radar". It involves an application of very low levels (1000 times less than a mobile phone) of microwave energy through the breast tissue. The foundation for tumour detection is a difference between the electrical properties of normal and malignant breast tissue. Normal breast tissue is largely transparent to microwave radiation while lesions, which contain more water and blood, scatters microwaves back towards the microwave source. The antenna picks up these reflected signals and they are analysed using a computer. A three dimensional image showing the location of the cancerous tissue can be obtained as the outcome of this signal processing [3].

Several research groups, including the University of Wisconsin-Madison and Dartmouth Collage of the US, University of Calgary in Canada, Technical University Denmark and others are currently doing research in this new area [2], [3], [5]. Similar activities have also been undertaken at the University of Queensland in Australia.

The majority of the developed microwave breast cancer detection prototypes are based on the synthesized pulse technique, in which a narrow pulse is generated in the frequency domain [6]. This synthesized signal is launched and received by an antenna which can be moved in small steps across a planar or curved area in front of an imaged object. At each location of the probe antenna, the received signal is processed with a Vector Network Analyser (VNA) which, by using its time domain capability, provides a suitable signal transformation from frequency to time or space domain. The measurement system is controlled from a PC. The collected data is gathered and processed by the PC to obtain a visual insight.

The conventional VNA with time-domain processing capability is handy to test and proof the concept of the microwave breast cancer detection system. However, because of its large size and high cost it may be precluded in a commercial breast cancer detection system.

The purpose of the work presented in this paper is to replace the conventional VNA by a low-cost alternative in the form of a wideband microwave six-port reflectometer. This paper is organised as follows, Section II, describes the experimental setup required for a microwave breast cancer detection system and provides results obtained using the conventional VNA with time-domain processing capability. Section III describes the design of a wideband 
reflectometer. Section IV and V compares two designs of the reflectometer, which use different types of couplers and power dividers. Section VI concludes the paper.

\section{EXPERIMENTAL SYSTEM}

The configuration of the prototype UWB radar system is shown in Fig. 1. The system consists of a $\Phi-Y$ circular cylindrical scanning platform which includes a turntable with a resolution of $22.5^{\circ}$ to support a breast phantom, and a mechanical scanning platform in the $\mathrm{Y}$ direction having resolution of $0.1 \mathrm{~mm}$.

The scanning platform supports a probe antenna which is in the form of an UWB tapered slot antenna. This antenna operates in the frequency range from $3.1 \mathrm{GHz}$ to 10.6 GHz [7]. A coaxial cable is used to connect the probe antenna to a microwave Vector Network Analyser (VNA), which is capable of measuring the full set of Sparameters of a 2 port network.

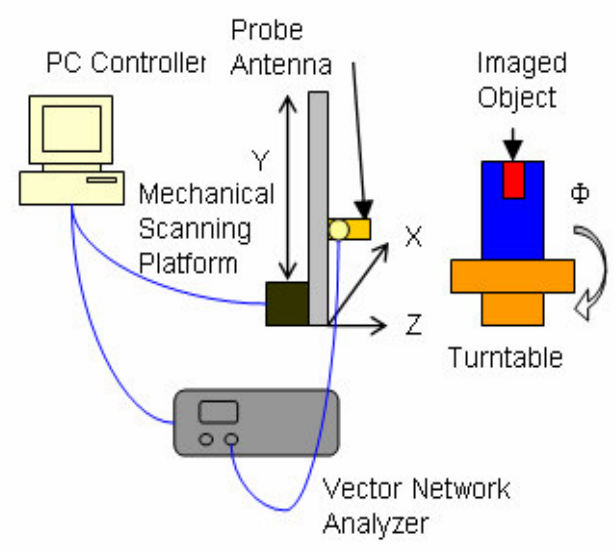

Fig. 1. The configuration of the microwave imaging system.

In the measurement procedure described in this paper, the VNA operates only in the reflection coefficient measurement mode. Prior to measurements, the system is calibrated over the frequency band from $3.1 \mathrm{GHz}$ to 10.6 $\mathrm{GHz}$ using a modified one-port calibration procedure. The coaxial short and shielded open circuit are used as in the standard calibration procedure. However the coaxial match termination is replaced by a load realized by the probe antenna radiating a microwave signal in free space [8]. We call this modified calibration procedure as Method A. By using Method A, undesired signals including internal reflections inside the probe antenna and at the antenna-air interface are either reduced or removed completely [8].

The measurement process is as follows. First, an area to be scanned is specified. The required information concerns the step size and a number of steps in the $\Phi-Y$ scanning platform.

At each $\Phi-Y$ probe location, the PC controller triggers the source in the Vector Network Analyser and 50 to 800 (depending on specifications) measurement points for reflection coefficient over the frequency band of interest are performed. After the frequency domain measurements of reflection coefficients are completed, they are converted to the time/space domain using IFFT.

Having obtained the frequency and time/space domain data for a given location, the results are stored in the PC and the probe is moved to a new position and the measurement procedure is repeated. The obtained data is then processed by the PC to create an image. Using false colours, the location of a target is identified in a colour distinctive from those representing other parts of the breast phantom.

The imaging capabilities of the above-described UWB radar system are carried out with respect to the experimental setup as shown in Fig.2.

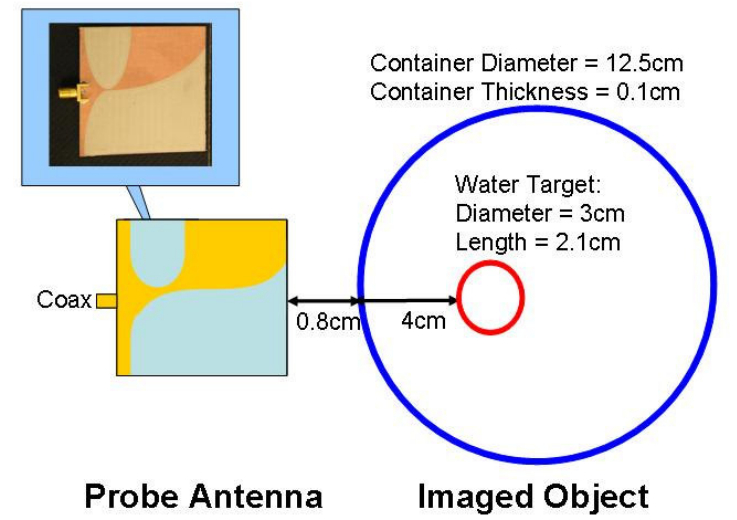

Fig. 2. Details of an experimental setup including an UWB tapered slot antenna and a plastic container with a target.

The breast phantom is formed by a circular cylindrical plastic container with a diameter of $12.5 \mathrm{~cm}$ with thickness of $1 \mathrm{~mm}$. The container is filled with vegetable oil with relative dielectric constant of 4 . The container and oil are used to represent the skin layer and breast tissue. A small plastic container filled with water (with relative dielectric constant of 80) representing the tumour is located close to the centre of the first plastic container. This phantom provides quite a good approximation to the actual healthy breast tissues and cancerous tumours, which have typical relative dielectric constants of 9 and 50, respectively [9].

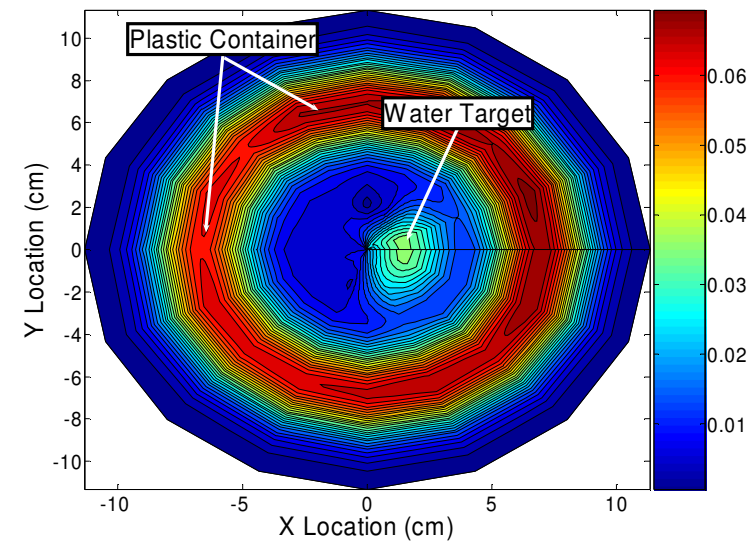

Fig. 3. The time domain reflection measurement result for plastic container filled with air, using the modified calibration technique. 
Fig.3 shows the imaging result when the system is calibrated using Method A. In these figure the boundaries of the plastic container and the location, size and shape of the water target are clearly observed. The measured diameter of the water target is approximately $3.3 \mathrm{~cm}$ and is quite close to the actual one of $3 \mathrm{~cm}$

\section{SiX PORT DESIGN}

Our next goal is to replace the VNA (of Fig. 1) by a six-port reflectometer composed of wideband couplers, dividers and scalar detectors. The configuration of the sixport which is investigated here is shown in Fig. 4.

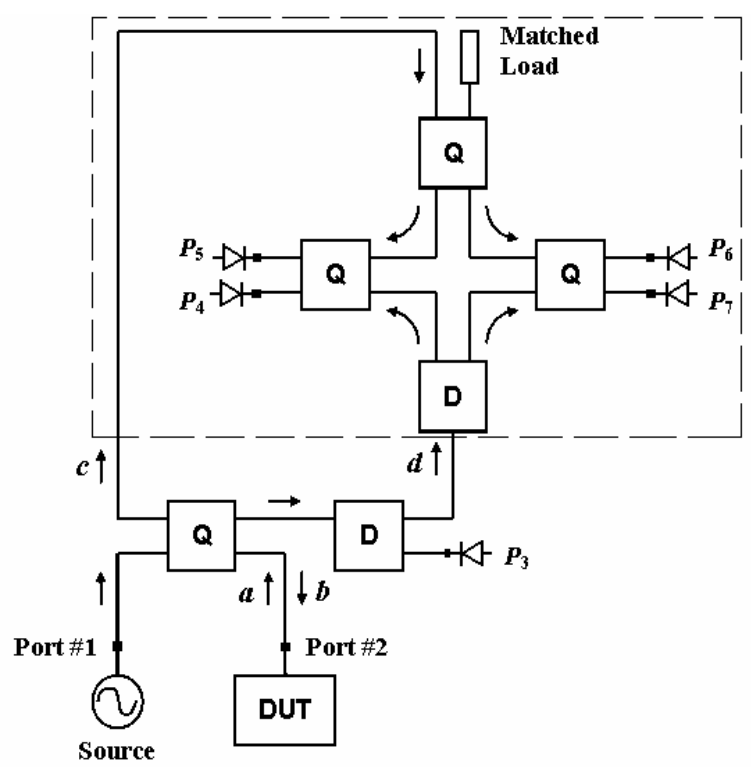

Fig. 4. Configuration of a six-port reflectometer with the realtime display capability.

This configuration, introduced in [10], offers real-time display of the measured complex reflection coefficient [11-13]. It includes quadrature hybrids (Q), and two-way power dividers (D). Also shown in Fig. 4 are scalar power detectors connected to ports 3-7, a microwave source connected to port 1 and a Device Under Test (DUT) connected to port 2. The part of the reflectometer enclosed by a broken line is called a Complex Ratio Measuring Unit (CRMU) or correlator. The Q hybrid outside the CRMU is employed to redirect a reflected signal, $a$ and an incident signal, $b$ at DUT to perform reflection-type measurements. Other circuits outside the CRMU can be used to redirect signals $a, b$ to perform transmission-type measurements. The divider D, outside the CRMU, terminated by a scalar detector at P3 is used to monitor the signal source power level. This signal can be treated as a reference signal. It can be used in a feedback loop to maintain a constant power level from the source.

Equation (1) defines the complex reflection coefficient as the ratio of the reflected signal from DUT, $a$ and the incident signal at DUT, $b$.

$$
\Gamma=a / b=\Gamma_{1}+j \Gamma_{2}
$$

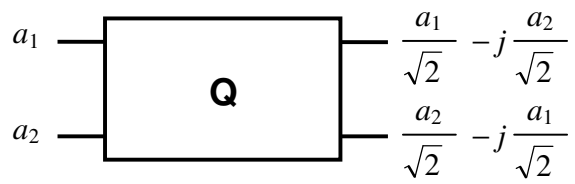

(a)

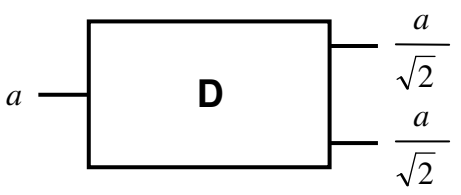

(b)

Fig. 5. The basic rules for the operation of (a) Quadrature hybrid (b) In-phase two-way power divider.

By considering the basic rule for operation of $\mathrm{Q}$ hybrid and D hybrid, as shown in the Fig. 5, the output signals at Port 3 to Port 7 of the reflectometer of Fig. 4 are given as shown in equations (2)-(6).

$$
\begin{aligned}
& S_{3}=-j \frac{b}{\sqrt{2}} \\
& S_{4}=-\frac{b}{2 \sqrt{2}}\left(\Gamma_{1}+j\left(\Gamma_{2}+1\right)\right) \\
& S_{5}=-j \frac{b}{2 \sqrt{2}}\left(\Gamma_{1}+j\left(\Gamma_{2}-1\right)\right) \\
& S_{6}=-\frac{b}{2 \sqrt{2}}\left(\left(\Gamma_{1}+1\right)+j \Gamma_{2}\right) \\
& S_{7}=j \frac{b}{2 \sqrt{2}}\left(\left(\Gamma_{1}-1\right)+j \Gamma_{2}\right)
\end{aligned}
$$

Assuming that the power detectors are identical and operate in the square-law region, the power values $P_{3}, P_{4}$, $P_{5}, P_{6}, P_{7}$ as measured by the detectors are given by (7):

$$
P_{i}=\left|S_{i}\right|^{2} \quad \text { where } i=3,4,5,6 \text { and } 7
$$

Using (7) in conjunction with (2-6), it can be proved that the complex reflection coefficient (1) is related to the measured powers at Port 3 to $7\left(P_{3}, P_{4}, P_{5}, P_{6}, P_{7}\right)$ by the following expression (8):

$\Gamma=\frac{a}{b}=\Gamma_{1}+j \Gamma_{2}=\frac{P_{6}-P_{7}}{P_{3}}+j \frac{P_{4}-P_{5}}{P_{3}}$

This expression indicates that for the reflectometer configuration of Fig. 4, the complex reflection coefficient, $\Gamma$, can be obtained by measuring voltages at the output of the square-law detectors. In the system controlled via PC, 
this task can be accomplished using a precision ADC card connected to the detectors. The PC monitor can be used to display $\Gamma$ in the polar or other formats.

\section{Two Reflectometer Configurations}

The six-port reflectometer of Fig. 4 can be realized in microstrip or stripline technology. Its performance will depend on the operation of the chosen Q and D hybrids. Two types of couplers and two types of power dividers in microstrip technology to construct $\mathrm{Q}$ and $\mathrm{D}$ hybrids are chosen to design two wideband reflectometers. In the first design, the 3dB Lange coupler [14] and the Gysel power splitter [15] are selected as the Q and D hybrids. In the alternative design, a $3 \mathrm{~dB}$ slot-coupled microstrip coupler and a 2-way Wilkinson power divider are chosen as the reflectometer's components. Although the alternative aim is to have the two designs for the $3.1-10.6 \mathrm{GHz}$ band, we have found that the first configuration has difficulty to meet this goal. Because of these reasons, the presented results for the first reflectometer are limited to the 4-8 $\mathrm{GHz}$ band.

\section{A. Lange Coupler and Gysel Power Splitter}

The configurations of the Lange coupler (consisting of 8 parallel strips with varying width, spacing and length) and the Gysel power splitter to form a wideband 6-port reflectometer (of Fig. 4) are presented in Fig. 6.

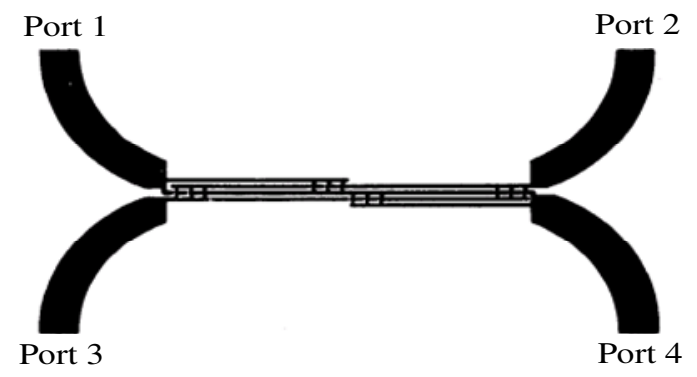

(a)

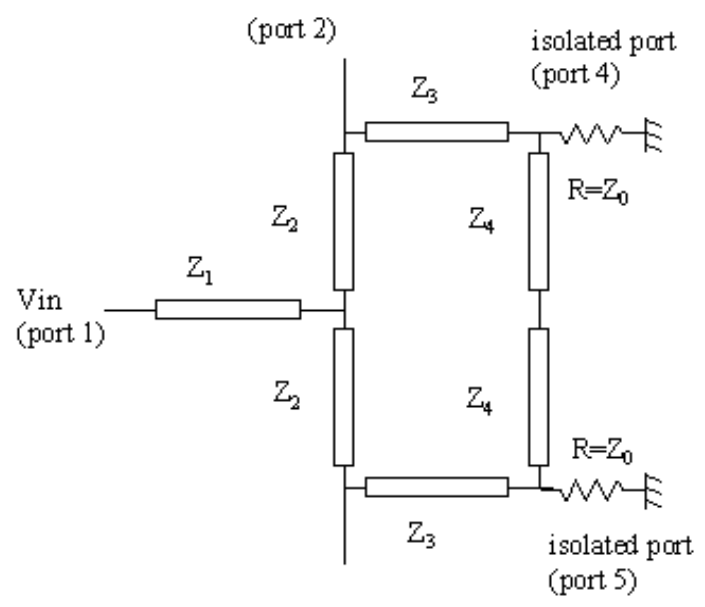

(port 3)

(b)

Fig. 6. Configurations of (a) Lange and (b) Gysel hybrids.
The performance of individual components is optimized by starting the design at the centre frequency of $6 \mathrm{GHz}$. The initial parameters are worked out using the design guidelines available from [14], [15].

It is worthwhile to mention that the selected Lange coupler needs wire crossover between very narrow strips (usually in the order of micro millimetre). In turn, the Gysel power divider is incapable of providing the required (3.1-10.6 GHz) multi-octave bandwidth performance.

In order to obtain the reflectometer with improved operational bandwidth new components are considered to form the second 6-port reflectometer.

\section{B. 3dB Slot-Coupled Microstrip Coupler and Wilkinson Power Divider}

In the alternative design, a 2-stage Wilkinson power divider, as shown in Fig. 7(a) [16] and a slot-coupled microstrip coupler, as shown in Fig. 7(b), are selected to form the reflectometer of Fig. 4.

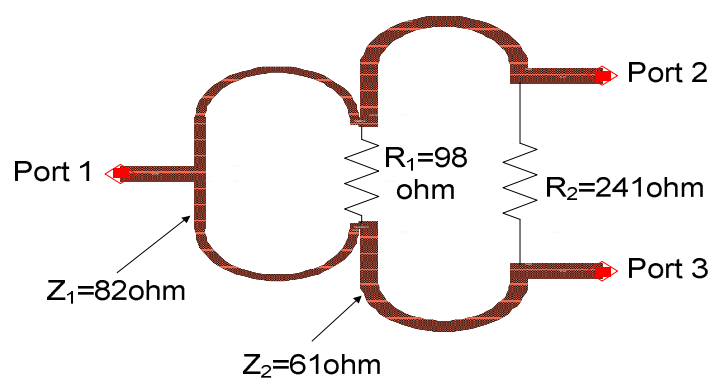

(a)

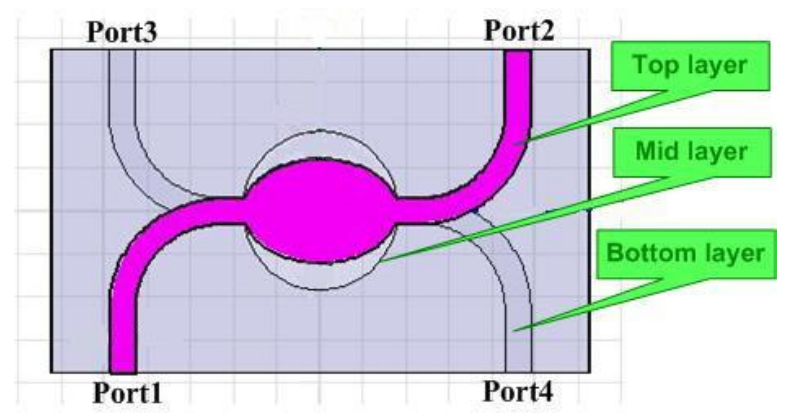

(b)

Fig. 7. Configurations of (a) Wilkinson power divider and (b) $3 \mathrm{~dB}$ slot-coupled microstrip coupler.

An explanation is required for the microstrip slotcoupled coupler. It is a new component, which has been recently proposed in [17]. The coupler has 3 conductor layers with 2 substrates between each layer. Upper and lower layer consist of identical elliptical shaped conductor patches with microstrip Ports 1-2 and 3-4, respectively. These two patches are coupled by an elliptical slot, which is in the middle conducting layer forming a common ground plane. The very important attribute of this coupler is its ability to offer a very wideband performance while preserving its very compact size. 


\section{Performance Comparison}

Using two sets of $\mathrm{Q}$ and $\mathrm{D}$ hybrids, two 6-port designs are accomplished. Their performance is assessed using Agilent ADS [18].

\section{A. First Reflectometer}

The first 6-port reflectometer uses the Lange coupler and the Gysel power splitter. Fig. 8 and 9 show the simulation results for specific DUTs such as short, open and match. Assuming the use of ideal square-law detectors, the reflection coefficient, $\Gamma$, was calculated using expression (9) that is analogous to (8):

$\Gamma=\frac{a}{b}=\Gamma_{1}+j \Gamma_{2}=\frac{\left|S_{61}\right|^{2}-\left|S_{71}\right|^{2}}{\left|S_{31}\right|^{2}}+j \frac{\left|S_{41}\right|^{2}-\left|S_{51}\right|^{2}}{\left|S_{31}\right|^{2}}$

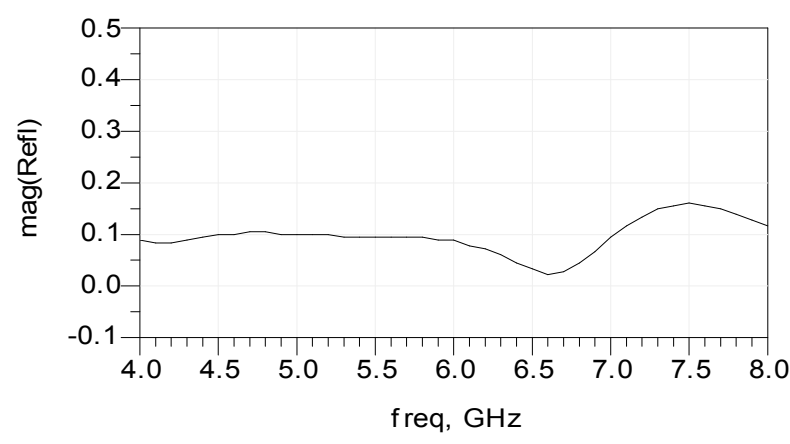

Fig. 8. Reflection coefficient determined by the first reflectometer for DUT in the form of matched load.

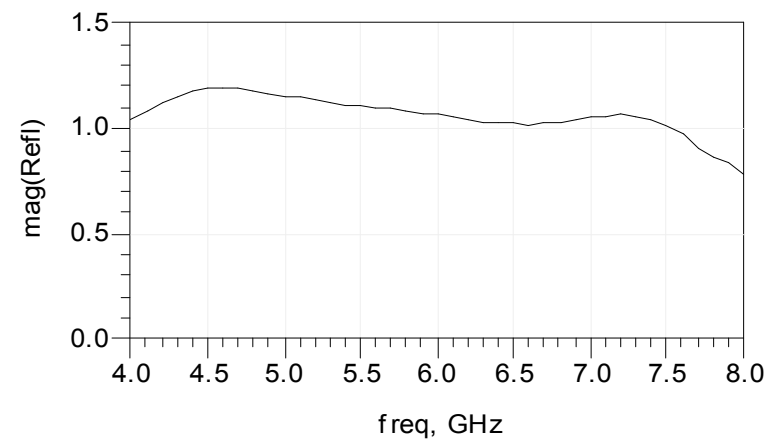

(a)

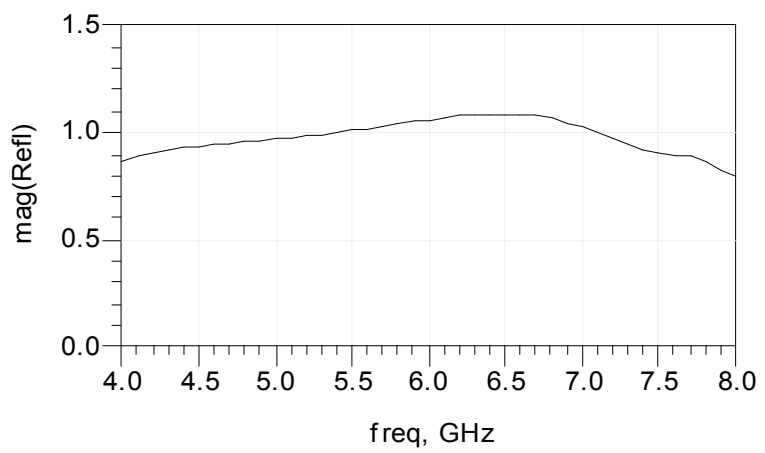

(b)

Fig. 9. Reflection coefficient determined by the first reflectometer for DUT in the form of (a) open, and (b) short circuit.
As observed in Fig. 8, the six-port reflectometer gives reflection coefficient, $\Gamma$ of $0.1 \pm 0.07$ across the $4 \mathrm{GHz}$ bandwidth when a matched load represent DUT. The observed deviation from the ideal case of zero is due to the non-ideal performances of individual components forming the reflectometer. Fig. 9(a)-(b) show the simulation results when DUT represents either a short or open circuit. The reflection coefficient, $\Gamma$ has a magnitude of $1 \pm 0.25$. The overall results in Fig. 8 and 9 indicate a reasonable performance of the six-port reflectometer over one octave (4-8 GHz) frequency band.

\section{B. Second Reflectometer}

The next simulations concern the performance of the second six-port reflectometer, which is formed by the 3 $\mathrm{dB}$ slot-coupled microstrip coupler and the Wilkinson power divider representing the $\mathrm{Q}$ and $\mathrm{D}$ hybrids in Fig. 4. As in the case of the first reflectometer, we assume the ideal square law operation of the detectors. The simulation results are shown in Fig. 10 and 11.

Fig. 10 presents the reflection coefficient, $\Gamma$, of the sixport reflectometer when the DUT is a matched load. Magnitude of $0.1 \pm 0.1$ is obtained from $3 \mathrm{GHz}$ to $10.6 \mathrm{GHZ}$

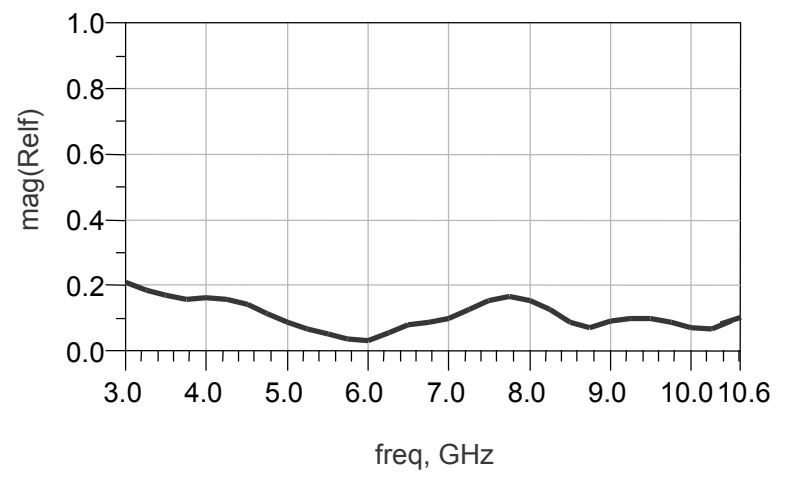

Fig. 10. Reflection coefficient determined by the second reflectometer for DUT in the form of matched load.

In turn, in Fig. 11 the reflection coefficient, $\Gamma$, with magnitude of $0.88 \pm 0.13$ is observed when DUT is in the form of either short or open circuit. The observed deviations are due to the non-ideal response of the components forming the reflectometer. When comparing the two reflectometer configurations, one finds that the use of the $3 \mathrm{~dB}$ slot-coupled microstrip coupler and the 2stage Wilkinson power divider results in the wider operational bandwidth, which is now $7.6 \mathrm{GHz}$.

The non-ideal operation of the two configurations of the reflectometer can be corrected by a suitable calibration procedure. For the investigated 6-port configurations, this task can be accomplished using the procedure involving 5 or 6 calibration standards, as described in [10-13].

Note that as the results for the DUT reflection coefficient obtained in the frequency domain need to be transformed to the time domain, the observed in Fig. 8-11 
deviations from actual values may be found tolerable.

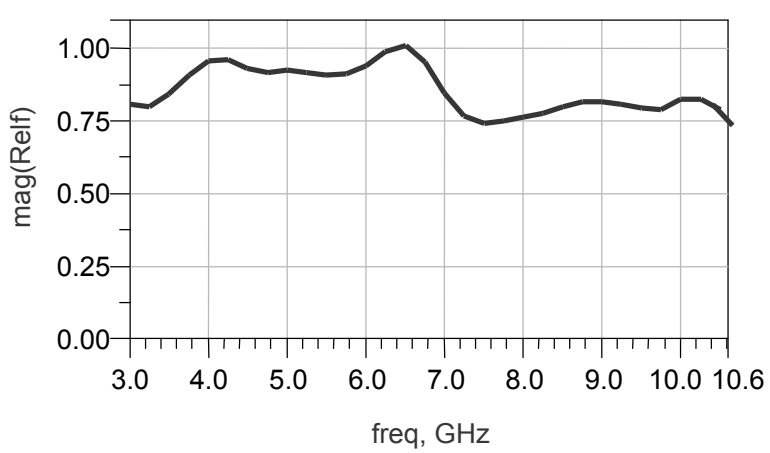

(a)

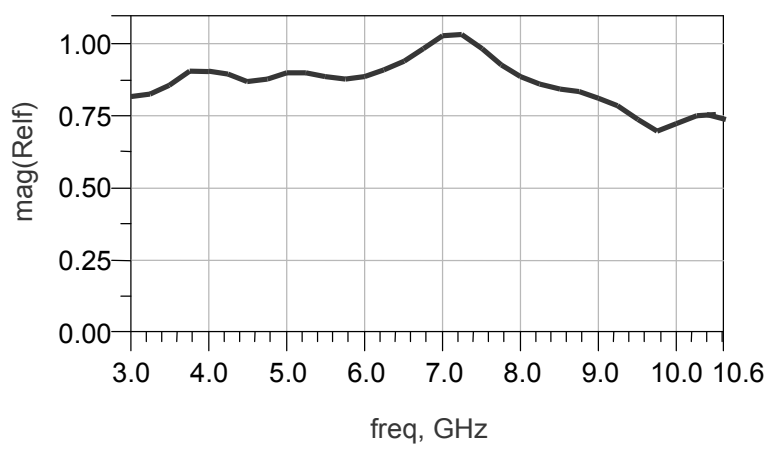

(b)

Fig. 11. Reflection coefficient determined by the second reflectometer for DUT in the form of (a) open or (b) short circuit.

As a result, one may find no need for a strict calibration of the presented reflectometers. Simple calibration procedures to remove small deviations between the actual and simulated values of the DUT reflection coefficient are currently investigated by our research team.

\section{CONCLUSION}

The design of two wideband microwave reflectometers for the purpose of inclusion in a microwave breast cancer detection system has been presented. In this system, a wideband frequency source is used to synthesize a narrow pulse via the step-frequency synthesis method to illuminate an imaged body. The reflectometer undertakes measurements in the frequency domain and the collected data is transformed into the time/space domain using an IFFT. A special configuration of a six-port reflectometer, which enables the measurement and display of the complex reflection coefficient in real-time by applying simple mathematical transformations of the measured voltages at the outputs of scalar (power) detectors has been described. One of the designed reflectometers uses the Lange coupler and the Gysel power splitter as its building blocks. The other one employs a slot-coupled microstrip coupler and a 2-stage Wilkinson divider, as its constituting components. The operation of the two reflectometers has been investigated with the use of Agilent ADS. It has been shown that the second reflectometer is capable to operate over an ultra wide frequency band, which is from 3.1 to $10.6 \mathrm{GHz}$.

\section{ACKNOWLEDGEMENT}

The authors acknowledge the financial support of the Australian Research Council via Grants DP0449996 and DP0450118.

\section{REFERENCES}

[1] Australian Institute of Health and Welfare \& Australasian Association of Cancer Registries (AACR), "Cancer in Australia 2001," AIHW cat. No.CAN23. Canberra: AIHW (Cancer Series no. 28), pp. 64-68, 2004.

[2] E.C. Fear, S.C. Hagness, P.M. Meaney, M. Okoniewski and M.A. Stuchly, "Enhancing breast tumor detection with near-field imaging," IEEE Micro, pp. 48-56, March 2002.

[3] E.C. Fear P.M. Meaney and M.A. Stuchly, "Microwave for breast cancer detection," IEEE Potentials, pp. 12-18, 2003.

[4] MARIBS study group, "Screening with magnetic resonance imaging and mammography of a UK population at high familial risk of breast cancer: A prospective multicentre cohort study," The Lancet, vol. 365, pp. 1769-78, 2005.

[5] Title: "Microwave imaging for breast-cancer screening" Available at:

http://www.emi.dtu.dk/research/afg/research/breastcancer_screeni ng.html

[6] D.A. Noon and M.E. Bialkowski., "An inexpensive microwave distance measuring system," Microwave and Optical Technology Letters, vol. 6, no. 5, pp. 287-292, April 1993.

[7] A. Abbosh, H.K. Kan and M.E. Bialkowski "A Compact UWB planar tapered slot antenna For use in a microwave imaging system" Microwave and Optical Techn. Letters, vol. 48, no.11, pp. 2212-2216, Nov. 2006.

[8] W.C. Khor and M.E. Bialkowski, "Investigations into cylindrical and planar configurations of a microwave imaging system for breast cancer detection" Proc. IEEE Antennas and Propagation Society International Symposium, pp. 263-266, Albuquerque, New Mexico, USA, Jul 2006.

[9] E. J. Bond, X. Li, S. C. Hagness, and B. D. Van Veen, "Microwave imaging via space-time beamforming for early detection of breast cancer," IEEE Trans. Antennas Propag., vol. 51, no. 8, pp. 1690-1705.

[10] C.A. Hoer, "A microwave network analyzer using two 6-port reflectometers", Microwave Symposium Digest, MTT-S International, vol. 77, issue 1, pp. 47-49, June 1977.

[11] S. Stuchly, M.E. Bialkowski, K. Caputa, and W. Guo, "Microwave level gauging system", Proc.10th Int. Microwave Conference, MIKON 94, Ksiaz, Poland, pp. 530-534, May 30-June 2, 1994.

[12] M.E. Bialkowski and A.P. Dimitrios, "A step-frequency six-port network analyser with a real-time display", $A E U$ Intl. Journal of Electronics and Communications, vol. 47, no. 3, pp. 193-197, 1993

[13] G.F. Engen, "An improved circuit for implementing the six-port technique of microwave measurements," IEEE Trans. Microwave Theory Tech., vol. MTT-25, no.12, pp. 1080-1083, Dec. 1977.

[14] J. Lange, "Interdigitated stripline quadrature hybrid", IEEE Trans. Microwave Theory Techn., pp. 1150-1151, Dec. 1969.

[15] U. Gysel, "A new N-way power divider/combiner suitable for high-power applications", Microwave Symposium Digest, MTT-S International, vol. 75, no. 1, pp. 116-118, 1975.

[16] M. L. Edwards, "Microwave \& RF circuits: analysis, design, fabrication, \& measurement", Sept. 2001.

[17] M.E. Bialkowski and A. M. Abbosh, "Design of a compact UWB 3dB coupler" submitted for publication, 2006.

[18] Agilent Technologies Advanced Design System 2002 - Passive Circuit Design Guide, Feb. 2002. 


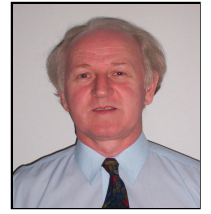

Marek E. Bialkowski (SM'88-F'03) was born in Sochaczew, Poland. He received the M.Eng.Sc. degree (1974) in applied mathematics and the Ph.D degree (1979) in electrical engineering both from the Warsaw University of Technology and a higher doctorate (D.Sc. Eng.) in computer science and electrical engineering from the University of

Queensland (2000)

$\mathrm{He}$ held teaching and research appointments at universities in Poland, Ireland, Australia, UK, Canada, Singapore, Hong Kong and Switzerland. At present he is a Professor in the School of Information Technology and Electrical Engineering at the University of Queensland. His research interests include antennas for mobile cellular and satellite communications, signal processing techniques for smart antennas, low profile antennas for reception of satellite broadcast TV programs, nearfield/far-field antenna measurements, electromagnetic modelling of waveguide feeds and transitions, conventional and spatial power combining techniques, six-port vector network analysers, and medical and industrial applications of microwaves. He has published over 450 technical papers, several book chapters and one book.

Prof. Bialkowski was a Co-recipient of a 2000 Harold A. Wheeler Applications Prize Honourable Mention for a paper published in the IEEE TRANSACtions on ANTENNAS AND PROPAgation in 1999. In 2002, he was elected an IEEE Fellow for contributions to the modelling, design, and testing of microwave guiding and radiating structures.

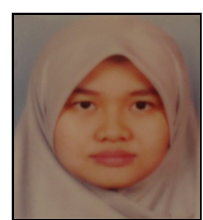

Norhudah Seman (S'06) was born in Johore, Malaysia. She received BEng degree (2003) in Electrical Engineering (Telecommunication) from the University Technology of Malaysia, Malaysia and M.Eng degree (2005) in Radio Frequency / Microwave Communications from The University of Queensland, Australia.

In 2003, she was employed as an engineer in Motorola Technology, Penang, Malaysia where she worked on RF and microwave components design and testing. Currently, she is a PhD student at The University of Queensland.

Her research interests concern the design of microwave circuits for biomedical and industrial applications, ultra wideband technologies and mobile communications.

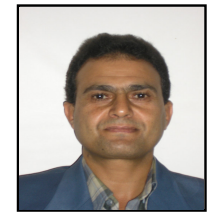

Amin M. Abbosh was born in Mosul, Iraq. He received the MSc in communications systems and $\mathrm{PhD}$ in microwave engineering in 1991 and 1996 respectively both from Mosul University. $\mathrm{He}$ worked as a Head of Computer \& Information Engineering Department, Mosul University, until 2003. In 2004, he joined the Griffith University and then the University of Queensland, Australia, as a Research Fellow. His research interests include antennas, radio wave propagation and microwave devices.

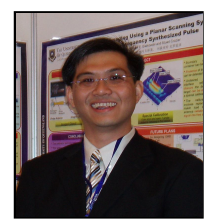

Wee Chang Khor ( $\left.\mathrm{S}^{\prime} 05\right)$ was born in Singapore. $\mathrm{He}$ received the B.Eng. degree in Electrical Engineering with Honours Class I from the University of Queensland in 2003.

Since 2004, he has been a Ph.D. student and a Research Assistant in the School of Information Technology and Electrical Engineering at the University of Queensland. His research interests include medical and industrial applications of microwaves. 